\title{
VALIDATION OF A NEW LONGITUDINAL TRAIN DYNAMICS CODE FOR TIME DOMAIN SIMULATIONS AND MODAL ANALYSES
}

\author{
NICOLA BOSSO ${ }^{1}$, MATTEO MAGELLI $^{2}$, NICOLÒ ZAMPIERI ${ }^{3}$ \\ Department of Mechanical and Aerospace Engineering, Politecnico di Torino, Italy
}

\begin{abstract}
Large speeds and axle-loads are required for modern freight trains, which can cause a big rise in in-train forces on wagon coupling elements for both tensile and compressive states, thus possibly leading to breaking of the coupling systems and to train derailments, respectively. Therefore, longitudinal train dynamics (LTD) simulations are a key tool for the prediction of the in-train forces and for the design of coupling and braking systems as well as for the optimization of the train composition. LTD simulations are typically carried out in time domain, to account for all the system non-linearities, mainly the hysteretic behaviour of the coupling system mechanical impedance characteristic. Although time domain simulations are a powerful tool to predict in-train forces considering all the system non-linearities, also frequency domain analyses can be useful to quickly compute the system dynamic behaviour. More in detail, modal analysis can provide important information on the system natural frequencies, so that the frequency content of the input forces can be checked to avoid the excitation of the system natural vibration modes.

The paper shows the development of a new efficient time domain simulation LTD code implemented in MATLAB, provided with a modal analysis post-processing routine. The code was validated on the four time domain simulation scenarios suggested by the international benchmark of LTD simulators, and a simplified modal analysis was also carried out on the same train configurations. The validation process highlighted that the new code provides stable numerical outputs with a good computational efficiency, while the modal analysis routine showed that the train eigenfrequencies can vary significantly according to the deflection, relative speed and loading state on each coupler.

Keywords: draft gear, dynamics modelling, long train simulation, LTD benchmark, modal analysis, train dynamics
\end{abstract}

\section{INTRODUCTION}

Modern freight trains are required to guarantee higher speeds and higher axle-loads to be a strongly competitive solution for goods transportation. Therefore, long trains composed of a huge number of vehicles exist, especially in the USA, China and Australia, where the coupler and draft gear system can transfer larger in-train forces with respect to the buffer-hook European solution [1]-[3]. The simulation of the longitudinal train dynamics (LTD) thus plays a crucial role for the prediction of the speed and position of each vehicle in the train composition during a specific trip on a selected route and especially of the in-train forces acting on each coupling system [4]. In fact, high tensile forces can lead to damages of the coupling systems and even to breaking of the train composition, while large compressive forces can cause train derailments [5],[6].

However, LTD simulation is a complex task since several nonlinear phenomena need to be included [7], mainly the hysteretic mechanical impedance of the coupling elements, which have different force-deflection characteristics for loading and unloading stages [8]. Therefore, LTD simulations are typically carried out only considering a single degree of freedom (d.o.f) in the running direction, with point masses, representing the vehicles, connected to

${ }^{1}$ ORCID:http://orcid.org/0000-0002-5433-6365

${ }^{2}$ ORCID:http://orcid.org/0000-0002-2962-7873

${ }^{3}$ ORCID:http://orcid.org/0000-0002-9197-1966 
each other by means of nonlinear spring damper elements, standing for the coupling systems. The effects of track slope and curvature are considered by adding to each vehicle a longitudinal resistant force. Moreover, an expression is needed also for the calculation of the ordinary resistant force, due to rolling resistances and aerodynamic drag. The model inputs also include the definition of the mechanical characteristics of the locomotive motors, the driving cycle, i.e., the notch level as a function of time or position of the leading locomotive, and optionally the braking command during the trip, i.e., the value of the pressure reduction in the brake main pipe as a function of time or position. Such models usually neglect the wheelrail adhesion phenomena [9] and the effects of traction/braking controls [10], which would require a high computational effort even in case optimized algorithms [11],[12] were used.

This strategy was suggested in the recently proposed international benchmark of LTD simulators [13],[14]. Since the aim of the benchmark was the comparison of the in-train forces calculated by different simulators in the same simulation scenarios, the simulation of the air brake system was neglected for the sake of computational efficiency, so that the only braking effort applied to the train was the dynamic braking of the locomotives. In fact, the bottleneck of single d.o.f LTD models is usually represented by the calculation of the nonlinear coupling forces. This calculation is typically performed according to three different approaches, as it can be inferred from the review paper by Wu et al. [15], i.e., i) fixed look-up table (LUT) models, ii) mathematical equations fitting the experimental hysteretic loops in the mechanical impedance characteristic and finally iii) white-box models, related to the physical and mechanical properties of the main components in the coupling system.

Obviously, more complex models, including the detailed simulation of the vertical and lateral dynamics of each or some vehicles [16]-[18] as well as of the air brake system [19]-[21], are witnessed in the literature; however, in this case, computational efficiency is a prime concern, and parallel or distributed computing techniques are essential to ensure good computing performances [22]-[24].

Since LTD involves several nonlinear phenomena, LTD simulations and analyses are typically carried out in time domain, thus requiring high computational times. However, frequency domain simulations, which need a system linearization to be performed, can be a useful tool for railway engineers to quickly investigate LTD problems in specific conditions. In fact, a preliminary knowledge of the natural frequencies of a long train can improve the choice of the proper time integration step to perform time domain simulations. Furthermore, in case experimental test campaigns are planned to be carried out on a specific train, the sampling rate of part of the instrumentation, such as longitudinal accelerometers and load cells, should be selected according to the train longitudinal natural frequencies.

Uyulan and Arslan [25] calculated the natural frequencies of a short train composed of a locomotive and nine wagons for low and high stiffness conditions and performed a spectral analysis of the coupling forces obtained from a time domain simulation in specific track sections, finding that peaks in the spectral analysis occurred for frequencies close to the system natural frequencies for both high and low stiffness values.

The paper shows the development and validation of a new time domain LTD simulator (LTDPoliTO) implemented in MATLAB, based on MATLAB vector logic for an efficient management of large arrays during the simulation, so that a high computational efficiency can be obtained [26],[27]. The validation of the new code is performed on the simulation scenarios suggested by the international benchmark. The simulator is provided with an optional postprocessing routine which allows performing a modal analysis of the train through a linearization of the coupling system characteristic in each time step of the solution. The modal analysis can be helpful to investigate whether external input forces, such as traction and braking forces, have a frequency content able to excite some of the train natural frequencies during the trip. 
The paper is organized as follows. The first section deals with the description of the code structure and implementation in MATLAB. The code strongly relies on MATLAB vector logic, which allows replacing traditional loop flows and Boolean statements, so that a higher computational speed can be achieved. Great focus is also given to the implementation of the modal analysis post-processing routine. Moreover, the simulation scenarios and the main input data used for the validation, which are extracted from benchmarking questions established in the international benchmark [13], are described in detail. The second section concerns the presentation of the results of the validation process, with great focus on both the numerical outputs and considerations of the code computational efficiency, as well as of the modal analysis, with emphasis on the train natural frequencies at the beginning of the simulation and on the evolution of the last natural frequency during the simulation. Finally, a last section deals with the conclusions on the presented paper and with suggestions for future works.

\section{CODE DEVELOPMENT}

\subsection{Definition of the model}

The need for the authors' research group to develop a new LTD simulator arose after a previous LTD model implemented in Simpack and joining the international benchmark showed significant numerical inefficiencies in the simulation of train configurations with many vehicles. The new code is implemented in MATLAB and is totally based on MATLAB built-in routines for large array indexing and management. At each time step during the simulation, the LTDPoliTO code does not require any explicit loop flow or Boolean statement. At this first stage of the code development, the code is specifically written for the simulation of the four train configurations suggested in the benchmark; however, the code can be easily adapted to any kind of train configuration and inputs.

The single d.o.f LTD model is described by a system of nonlinear ordinary differential equations (o.d.e). For a train composed of $N$ vehicles, at each time step $t$, it is easy to demonstrate that the algebraic system of eqns. (1a)-(1d) needs to be solved, where $[M]$ is the $2 N \times 2 N$ mass matrix of the system, $\boldsymbol{y}$ is the $2 N$ elements array containing the solution of the problem, $\underline{\boldsymbol{y}}^{\prime}$ is the $2 \mathrm{~N}$ elements array containing the time derivative of the solution and finally $\boldsymbol{f}=\boldsymbol{f}(t, \underline{\boldsymbol{y}})$ is the function relating the solution to the dependant variables of the problem.

In eqns. (1a)-(1d), $j$ refers to the $j$ th vehicle in the train composition, with the vehicles numbered in ascending order from the tail-end wagon to the leading locomotive, $m_{v}$ is the vehicle mass, $\dot{x}$ and $\ddot{x}$ refer to the vehicle speed and acceleration, respectively, $F_{T / D B}$ is the force due to traction or dynamic braking, $F_{\text {Res }}$ is the sum of all resistant forces, and finally $F_{C}$ is the force on the coupling system.

$$
[M] \underline{\boldsymbol{y}^{\prime}}=\underline{\boldsymbol{f}}(t, \underline{y})
$$

$$
[M]=\left[\begin{array}{cccccc}
1 & & & & & \\
& \ddots & & & & \\
& & 1 & & & \\
& & m_{v, 1} & & \\
& & & \ddots & \\
& & & & m_{v, N}
\end{array}\right] .
$$




$$
\left.\begin{array}{c}
\underline{\boldsymbol{y}}^{\prime}=\left\{\begin{array}{c}
\dot{x}_{1} \\
\vdots \\
\dot{x}_{N} \\
\ddot{x}_{1} \\
\vdots \\
\ddot{x}_{N}
\end{array}\right\} . \\
\dot{x}_{1} \\
\vdots \\
\dot{x}_{N} \\
F_{T / D B, 1}-F_{R e s, 1}+F_{C, 1} \\
\vdots \\
F_{T / D B, j}-F_{R e s, j}+F_{C, j}-F_{C, j-1} \\
\vdots \\
F_{T / D B, N}-F_{R e s, N}-F_{C, N-1}
\end{array}\right\} .
$$

The sum of all resistant forces $F_{\text {Res }}$ acting on each vehicle is the sum of three terms, i.e., the resistant force due to rolling resistances $F_{R R}$, the resistant force due to track curvature $F_{R C}$ and the resistant force due to track grade $F_{R G}$, see eqns. (2)-(4), where $m_{a x}$ is the vehicle axleload, $Q$ is the aerodynamic resistant factor, which is considered equal to 3.2 for the leading locomotive and equal to 1 for all other vehicles in the train composition, $R_{c}$ is the curve radius (m) and $i_{G}$ is the track grade (\%o). Please note that in eqns. (2)-(4), the unit for masses is tonne and the unit for speed is $\mathrm{km} / \mathrm{h}$. Of course, other expressions are witnessed in the literature for the calculation of the ordinary resistant forces and of the resistant force due to track curves [28],[29], and work is already being carried out to allow the LTDPoliTO user to select the preferred expression for each vehicle in the train [30].

The resistant force due to pneumatic braking is neglected in accordance with the international benchmark; however, future upgrades to the code will deal with the implementation of a dedicated module for the computation of the air brake forces.

$$
\begin{gathered}
F_{R R}=Q m_{v}\left(2.943+\frac{89.2}{m_{a x}}+0.0306 \dot{x}+\frac{0.122 \dot{x}^{2}}{m_{v}}\right) . \\
F_{R C}=m_{v} \frac{6116}{R_{C}} . \\
F_{R G}=m_{v} g i_{G} .
\end{gathered}
$$

The locomotive force due to traction or dynamic braking is calculated from the mechanical characteristic of the locomotive motors as a function of vehicle speed and notch level, while the forces acting on each coupling system are computed using a LUT strategy, with a smoothing transition between the loading and unloading curves as a function of the relative 
speed, according to the method suggested by Zhang et al. [31], see eqns. (5a)-(5c). In eqns. (5a)-(5c), $\delta x$ and $\delta v$ refer to the coupling system deflection and relative speed, respectively, $F_{L}$ is the loading force, $F_{U}$ is the unloading force, $F_{m}$ and $F_{a}$ are auxiliar quantities calculated from the loading and unloading forces and finally $v_{\varepsilon}$ is a threshold value for the smoothing transition management.

$$
\begin{gathered}
F_{C}(\delta x, \delta v)=\left\{\begin{array}{r}
F_{L}(\delta x),|\delta v| \geq v_{\varepsilon} \wedge \delta x \cdot \delta v \geq 0 \\
F_{U}(\delta x),|\delta v| \geq v_{\varepsilon} \wedge \delta x \cdot \delta v<0 \\
F_{m}+F_{a} \frac{\delta v}{v_{\varepsilon}},|\delta v|<v_{\varepsilon}
\end{array}\right. \\
F_{m}=\frac{F_{L}(\delta x)+F_{U}(\delta x)}{2} . \\
F_{a}=\frac{\left|F_{L}(\delta x)-F_{U}(\delta x)\right|}{2} .
\end{gathered}
$$

Fig. 1 shows the flow chart of the LTDPoliTO code implemented in MATLAB, which is composed of four main stages and an additional post-processing optional routine for modal analyses. The first stage of the code deals with loading the input text files, which define the track features (curvature and grade), the driving cycle, the mechanical characteristics of the locomotives and the hysteretic mechanical impedance of the coupling systems. Then, the pre-processing routine is activated, which defines the train configuration, the initial speed and position of each vehicle and the solver options. Moreover, in this stage, the input text files are re-interpolated with user-defined fixed steps, to allow a straightforward indexing throughout the simulation, with no need for loop flows or Boolean conditions. Then, the o.d.e system is numerically integrated by the variable step-size predictor corrector stiff solver ode $15 \mathrm{~s}$ [32],[33], with a pre-definition of the system Jacobian sparsity pattern, allowing for a big speed up of the computation in failed time steps. Once the o.d.e system is solved, the position and speed of each vehicle throughout the simulation are known, and a post-processing routine calculates the deflection, relative speed and force on each coupler in all time steps. All these outputs can be saved in three different binary files for storage and data analysis purposes. Finally, the optional modal analysis routine can be called, which performs a linearization of the coupling system mechanical impedance characteristic, so that a stiffness matrix of the system as well as eigenvalues and eigenvectors of the train can be computed at each time step.

\subsection{Definition of the case study}

The validation of the code was performed on the four simulation scenarios suggested by the international benchmark, which refer to four different train configurations, composed of two different types of wagons and two different types of locomotives (see Tables 1 and 2). The total number of d.o.fs increases from trains 1 to 3 , while train 4 is composed of the same number of vehicles as train 3, but it features a different arrangement. Being $N$ the total number of vehicles in a simulation scenario, the train configuration is defined in the new code by means of a $1 \mathrm{D}$ array containing $N$ integers in the range 1-4. However, work is currently in progress to adapt the code to any possible train configuration, built using many different types of wagons and locomotives. 


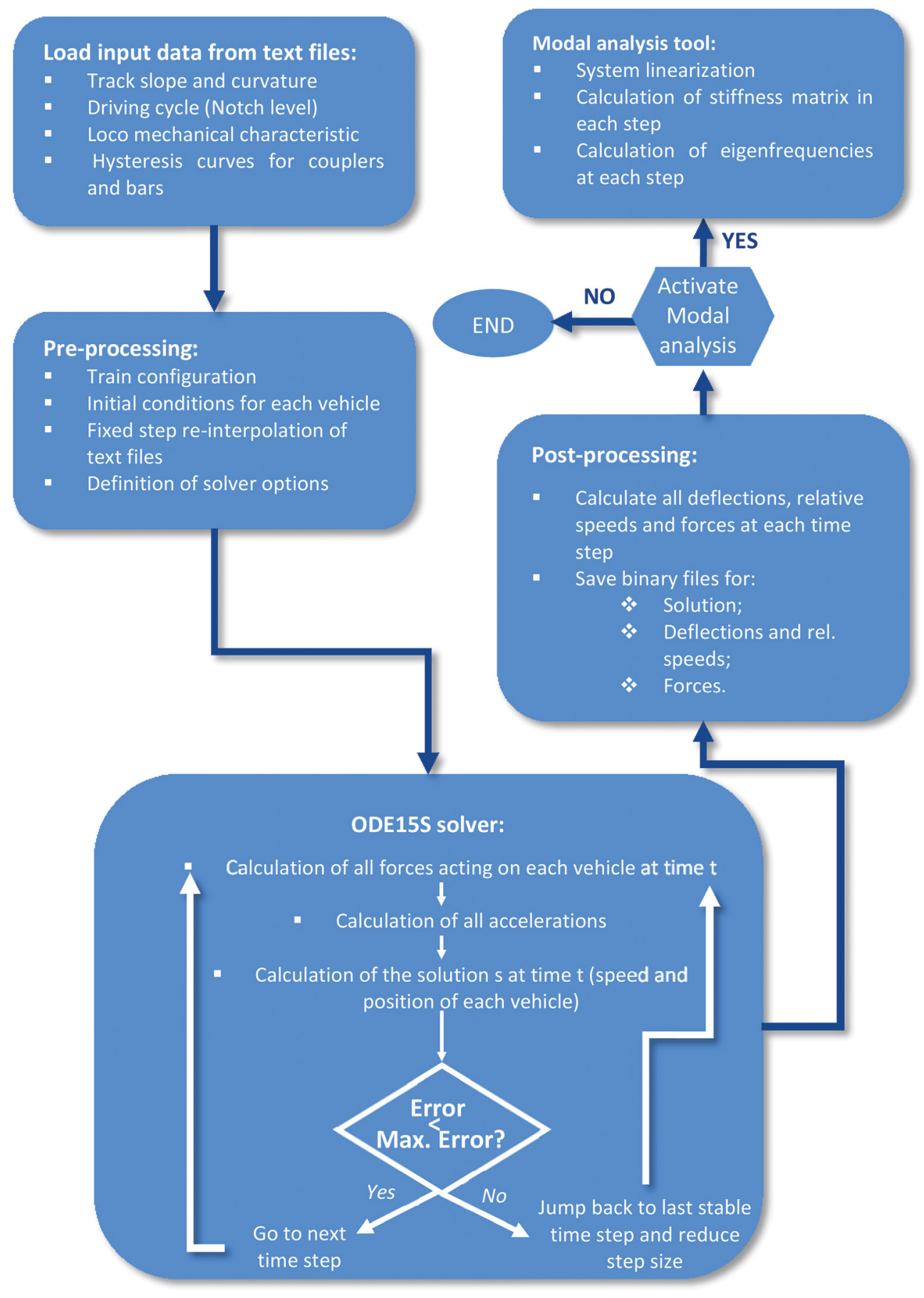

Figure 1: LTDPoliTO flow chart. 
The benchmark input data also include the mechanical characteristics of the two locomotives, the values of curvature and grade of a realistic track and the driving cycle for each simulation scenario. Moreover, a force-deflection cross-plot for a single draft gear was provided to all participants, who however could choose the preferred modelling strategy of the coupling elements according to their previous experience (see Fig. 2). Two types of coupling elements are considered in the benchmark, i.e., couplers and drawbars, having the same mechanical impedance characteristic apart from a total slack of $10 \mathrm{~mm}$ for the coupler. In all four train configurations, wagons are connected using bars to form a wagon pair, and each wagon pair is connected to the adjacent ones with couplers, while locomotives are connected to all other vehicles with couplers.

The next section will deal with the results of the code validation process, which was performed comparing the outputs calculated from LTDPoliTO with those obtained from the other benchmark participants. At the same time, great focus was given during the validation process to the removal of the numerical divergences that occurred with the previous LTD Simpack model. The validation also concerned the computational speed of the code with respect to other simulators which joined the benchmark competition. All simulations were performed with the following solver parameters: i) relative tolerance of $1 \mathrm{e}-06$, ii) absolute tolerance equal to $1 \mathrm{e}-07$ and iii) maximum step of $0.1 \mathrm{~s}$, while the relative speed threshold value for the logic switch, see eqns. (5a)-(5c), was set to $1 \mathrm{e}-03 \mathrm{~m} / \mathrm{s}$.

Moreover, at the end of the validation process, a new simulation was run for each train configuration to evaluate the eigenfrequencies of each train throughout the simulations, using

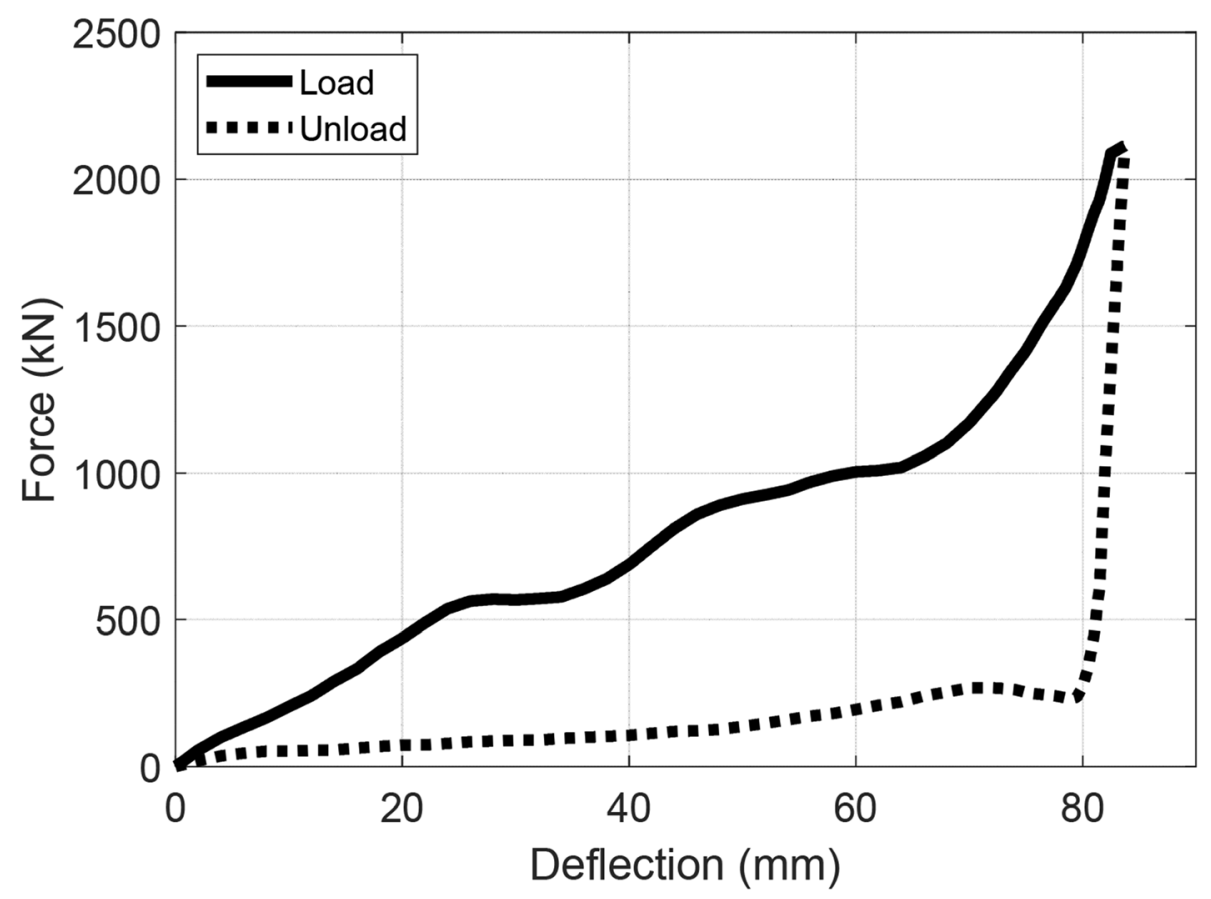

Figure 2: Force-deflection characteristics of a single draft gear provided by the benchmark. 
Table 1: Description of the four train configurations suggested in the benchmark.

\begin{tabular}{ll}
\hline Simulation scenario & Train configuration \\
\hline Train 1 & 2 Loco \#1 + 50 Wagon \#1 \\
Train 2 & 2 Loco \#1 + 50 Wagon \#1 + 2 Loco \#1 + 50 Wagon \#1 \\
Train 3 & 3 Loco \#2 +240 Wagon \#2 \\
Train 4 & 2 Loco \# $2+120$ Wagon \#2 + Loco \#1 + 120 Wagon \#2 \\
\hline
\end{tabular}

Table 2: Main parameters of the four vehicle types presented in the benchmark.

\begin{tabular}{lllll}
\hline Vehicle type & Axle-load (tonne) & Axle numbers & Total mass (tonne) & Vehicle length $(\mathrm{m})$ \\
\hline Loco \#1 & 22.33 & 6 & 134 & 22.95 \\
Loco \#2 & 32.50 & 6 & 195 & 24.35 \\
Wagon \#1 & 32.00 & 4 & 128 & 15.00 \\
Wagon \#2 & 40.00 & 4 & 160 & 11.00 \\
\hline
\end{tabular}

the modal analysis post-processing routine. These investigations were performed on time domain simulation run using piecewise linear mechanical impedance characteristic for both couplers and bar in loading and unloading stages. This choice allowed to simplify the calculation of the system stiffness matrix in all four simulations and to avoid negative stiffness values, which would lead to complex eigenvalues. The stiffness matrix is computed in each time step, assuming that when the force acting on a coupler is equal to either the loading or the unloading force, then it is treated as a fully elastic force, and the calculation of the coupler stiffness is straightforward. On the other hand, when the coupler is in switching conditions between the two curves, the stiffness is calculated as the mean stiffness between the loading and unloading curves, according to eqns. (5a)-(5c). Of course, this is not perfectly accurate since the force stored in the LUTs is the total force given by the draft gear, which has both a spring and a damping component. Nevertheless, if a LUT model is used, the two terms are difficult to be isolated. In future works, a fitting equation model could be developed to simplify the calculation of the coupling system stiffness and to distinguish between the elastic and the damping contributions in loading and unloading conditions. However, in the authors 'opinion, this limitation can be accepted at this first stage of the code development.

\section{RESULTS}

The code validation was carried out considering the following main output quantities defined in the benchmark results paper (see Table 3):

- The maximum and mean speed values, calculated considering all vehicles and time steps.

- The largest in-train force and the corresponding position, i.e., the maximum value of force for both tensile and loading states, considering all time steps and all coupling systems.

- The mean of the maximum values of force registered on all coupling systems among all time steps for both tensile and compressive states.

- The values of the maximum deflection on a selected coupling position for both tensile and compressive states. 
According to the benchmark rule, in this section, negative values are used for tensile states, while positive values refer to compressive conditions, and coupling elements are numbered in ascending order starting from the one on the rear of the leading locomotive.

As it can be inferred from the comparison of Table 3 with the output results presented in the benchmark results paper, a good accordance exists between LTDPoliTO and the other simulators, concerning the speed values, the largest in-train forces and the mean in-train force. On the other hand, LTDPoliTO tends to compute larger deflections of the coupling elements, and this could be related to differences in the switching transitions and in the slack distribution between tensile and compressive states. For the sake of clarity and readability, Table 4 shows the results computed by the simulators joining the benchmark in the third simulation scenario, in which the original PoliTO Simpack code produced numerical instabilities, while the new MATLAB LTDPoliTO code computes numerically stable outputs.

Fig. 3 presents the force on the selected coupler position for all four simulation scenarios computed by LTDPoliTO and by the previous Simpack model, which produced numerically unstable values for trains 3 and 4 . On the contrary, the output calculated with LTDPoliTO is numerically stable and no divergences occur.

Focusing on the computational efficiency of LTDPoliTO, the new code proved to be the second fastest simulator in all four simulations, except for TABLDSS, as it is shown in Fig. 4, where the speed 1 value is plotted. This value is calculated as the ratio between the milliseconds of wall time and the train operational time in seconds: if the speed 1 value is equal to 1,000 , then the simulation runs in real time. A fair comparison of the simulators in terms of computational efficiency however would require all participants to run their codes on the same hardware platforms and OS. The simulations presented in this paper were carried out on a notebook with these main features: Windows 10 Pro OS, Intel i7 CPU and 16 GB of internal memory. Nevertheless, the new LTDPoliTO simulator proved to be a fast and numerically stable code.

Once the code was validated against the outputs of the other benchmark results, a new set of simulations was performed using piecewise linear characteristics for the loading and unloading curves of bars and couplers. Assuming that the force on the loading and unloading curves is fully elastic, the equivalent stiffness for a pair of couplers and bars is shown in Fig. 5. The new set of simulations gave similar results with respect to those obtained using the fully nonlinear characteristics. At the end of the simulations, the modal analysis routine was activated to compute the train eigenfrequencies during the simulated operation time for each train configuration.

Table 3: LTDPoliTO results for the four benchmark train configurations.

\begin{tabular}{cccllllllll}
\hline & \multicolumn{3}{c}{ Speed $(\mathrm{km} / \mathrm{h})$} & \multicolumn{2}{l}{$\begin{array}{l}\text { Largest in-train force }(\mathrm{kN}) \\
\text { @ coupler pos. }\end{array}$} & \multicolumn{2}{l}{$\begin{array}{l}\text { Mean in-train } \\
\text { force }(\mathrm{kN})\end{array}$} & \multicolumn{3}{l}{$\begin{array}{l}\text { Max. deflection }(\mathrm{mm}) \\
\text { @ selected coupler pos. }\end{array}$} \\
\hline \multicolumn{2}{rl}{ Train } & & Max. & Mean & Tens. & Comp. & Tens. & Comp. & Pos. Tens. & Comp. \\
\hline 1 & 87.30 & 65.97 & $-565 @ 2$ & $338 @ 2$ & -336 & 195 & 10 & -64.56 & 45.07 \\
2 & 89.40 & 64.55 & $-769 @ 54$ & $407 @ 54$ & -444 & 263 & 61 & -89.22 & 48.05 \\
3 & 81.83 & 44.79 & $-1,636 @ 3$ & $1,008 @ 144$ & $-1,166$ & 620 & 147 & -169.56 & 129.00 \\
4 & 81.80 & 44.99 & $-1,309 @ 123$ & $867 @ 122$ & -932 & 501 & 146 & -161.71 & 96.35 \\
\hline
\end{tabular}




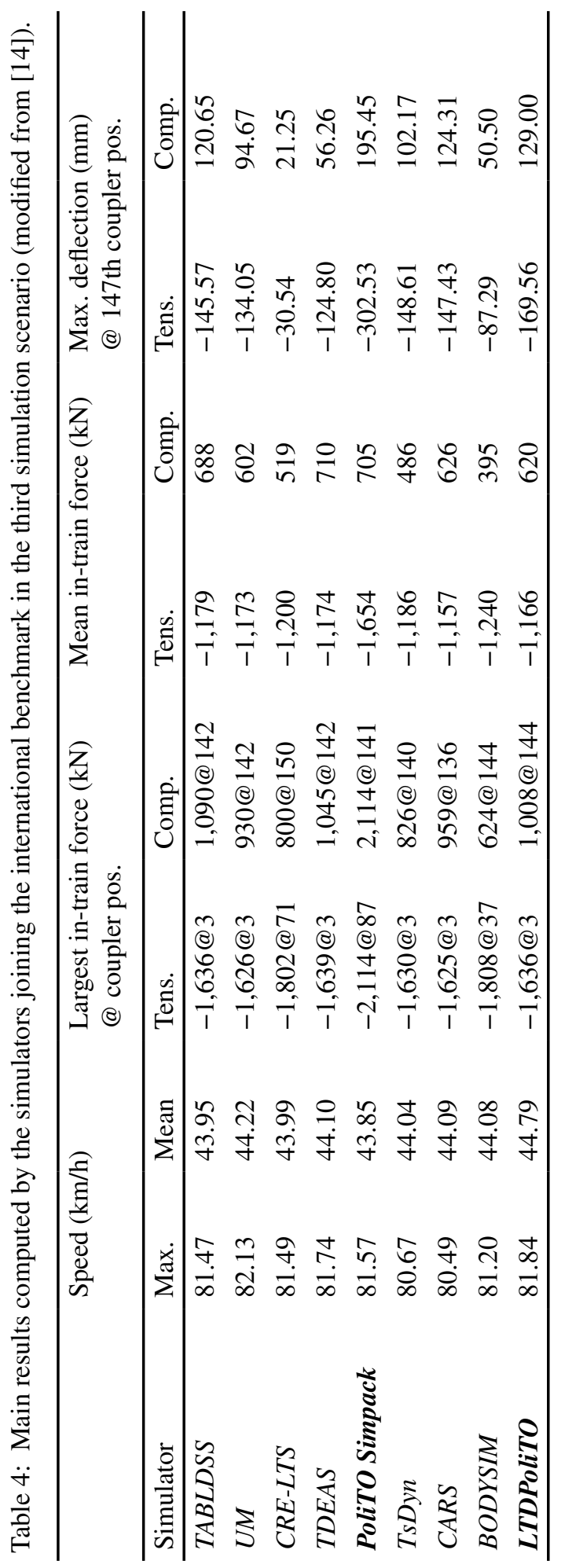



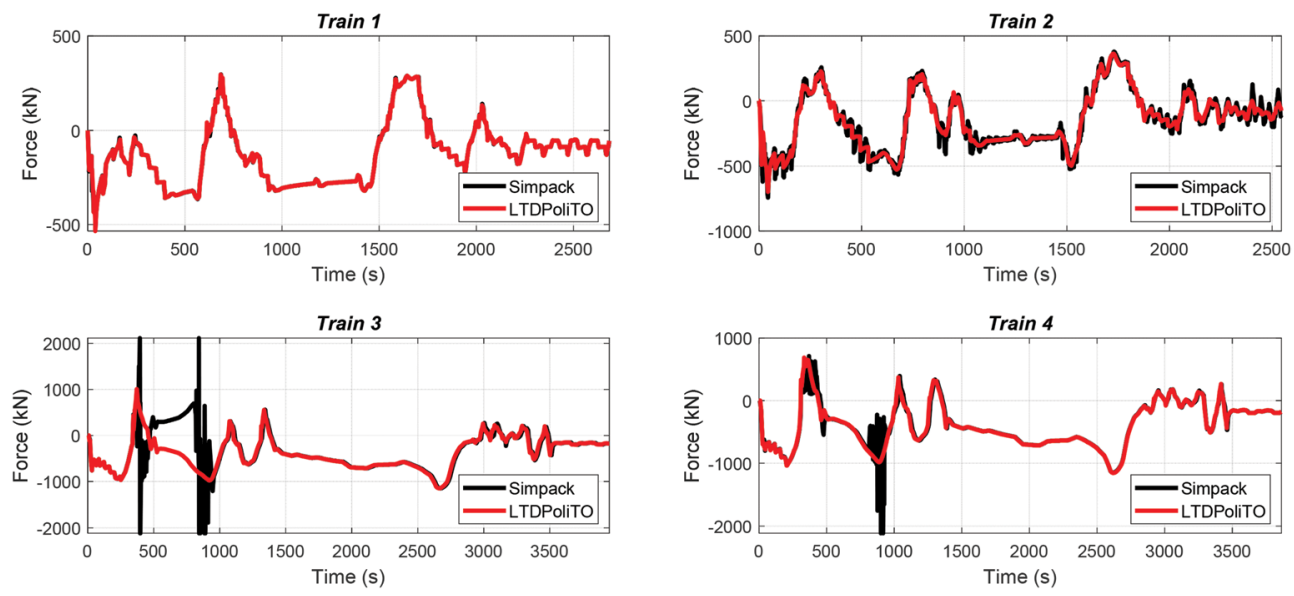

Figure 3: Comparison of output results from LTDPoliTO and previous Simpack model for the selected coupler position on all four train configurations.

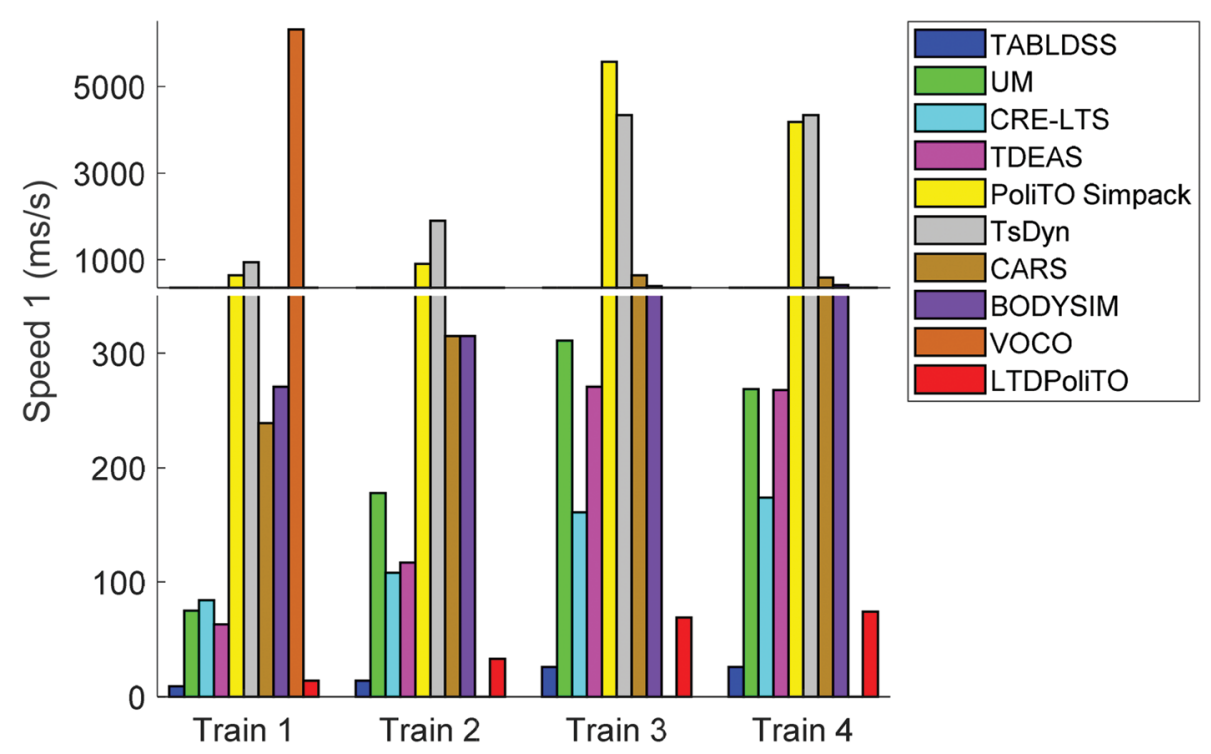

Figure 4: Speed 1 value for all four simulation scenarios.

Fig. 6 shows the eigenfrequencies of each train at the beginning of the simulation. For each configuration scenario, there are several modes with an eigenfrequency equal to 0 , due to the rigid motions that are allowed by the slack of the couplers. For instance, in train 1, there are 25 wagons pair and 2 locomotives. Each wagon pair has a null eigenvalue due to the rigid motion and an eigenvalue equal to $2 k_{B, m} / m_{v}$, where $k_{B, m}$ is the bar stiffness and $m_{v}$ is the vehicle mass, while the two locomotives have null eigenvalues since they are connected to all other vehicles with couplers. The bar stiffness is the mean value between the loading and unloading stiffness at $t=0$, and it is estimated equal to $6.16 \mathrm{kN} / \mathrm{mm}$. Similar considerations are also valid for the other train configurations. 


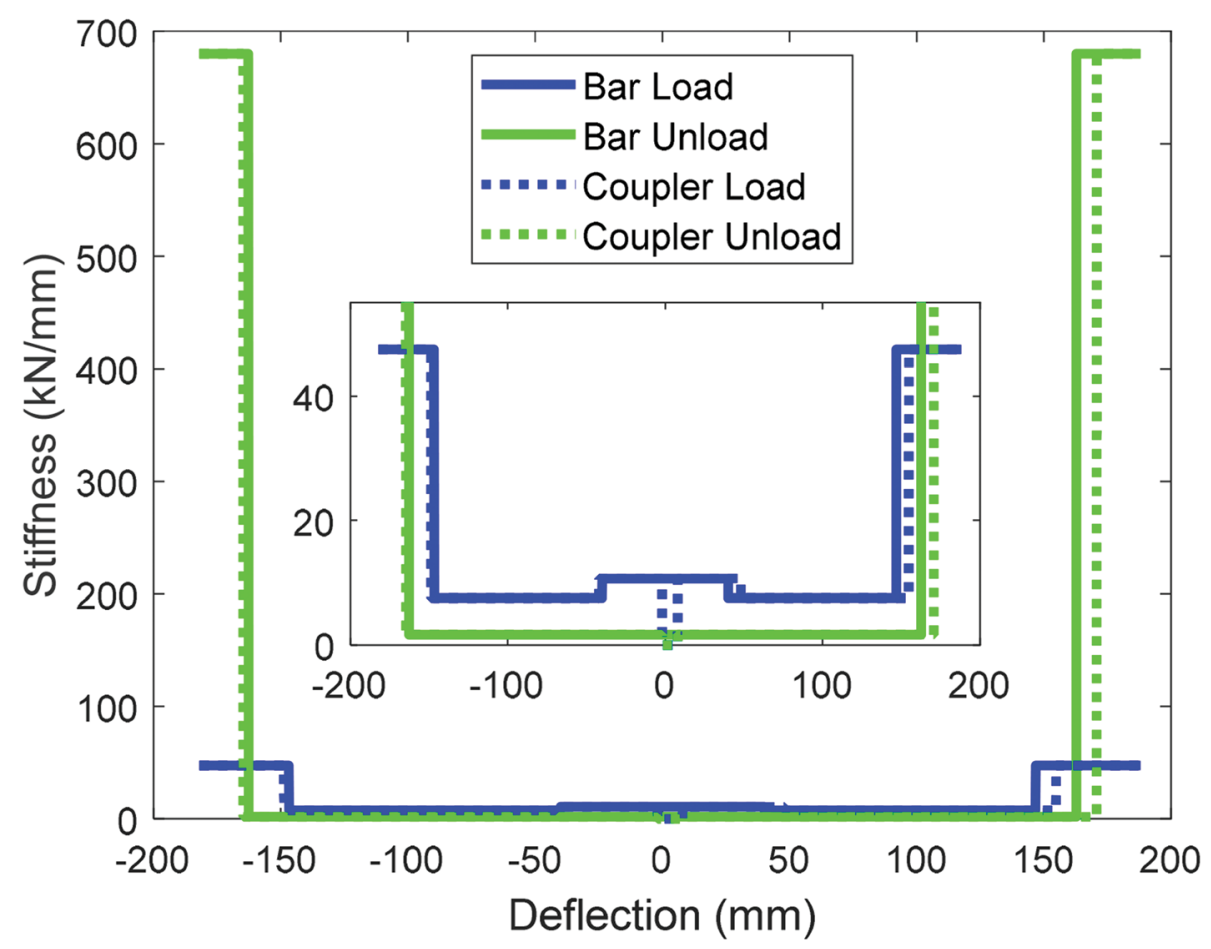

Figure 5: Stiffness of bar and coupler with the piecewise linear mechanical impedance.
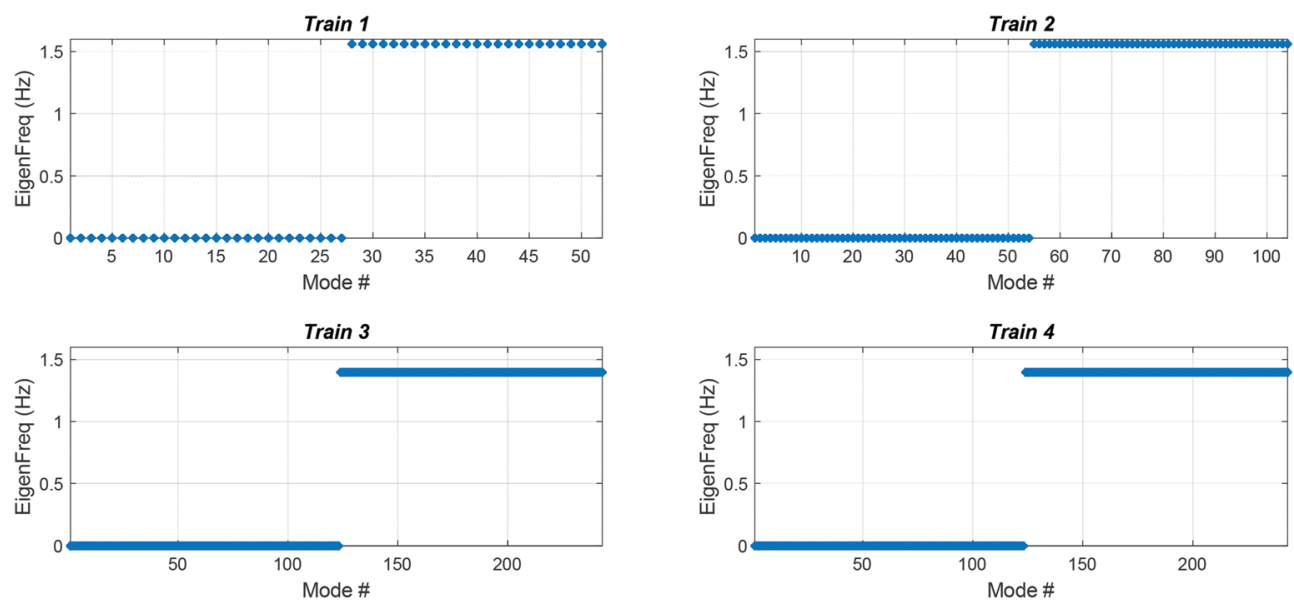

Figure 6: Eigenfrequencies at $t=0$ for all four train configurations.

Fig. 7 presents the evolution of the largest natural frequency of train 3 during the simulation. The deflection and stiffness for five coupler positions starting from the rear of the third locomotive are also shown in the same plot. A great variation of the maximum system natural frequency can be observed during the whole trip, due to changes in stiffness and loading states 

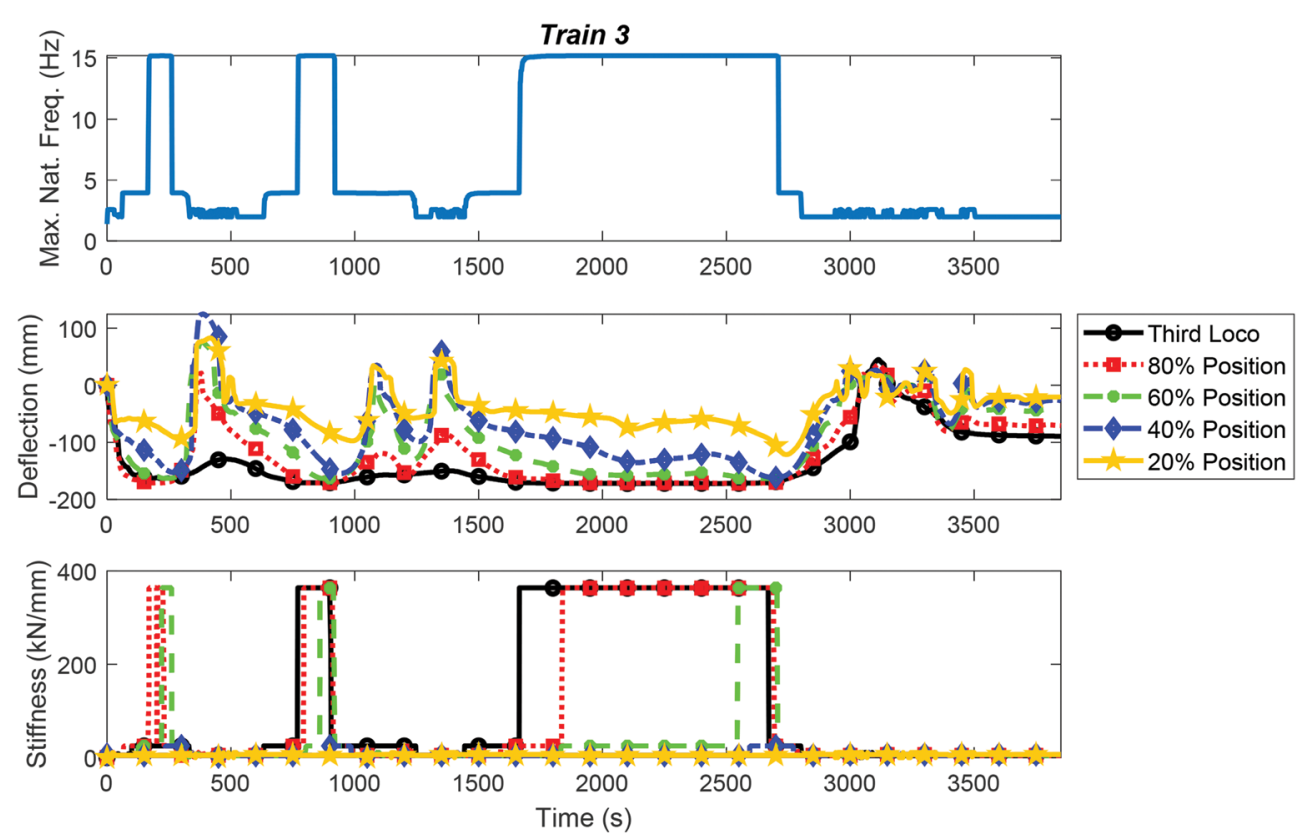

Figure 7: Evolution of largest eigenfrequency, coupler deflections, and stiffnesses during the whole third simulation scenario.

caused by the vehicle running on the line. Since the stiffness matrix of the system depends on the stiffness of each coupling element, which is a function of the relative speed and deflection of the coupler itself, it is shown that different values of maximum eigenfrequency can be obtained. Higher values of the train largest eigenfrequency are obviously computed when the deflection and loading state on several couplers are such that the stiffness value on such couplers becomes very high. Similar results can also be found on the other train configurations.

\section{CONCLUSIONS}

A new LTD code, named LTDPoliTO, is presented in the paper, which can perform both time-domain simulations, to consider all the non-linearities of coupling elements, and modal analyses, using a dedicated post-processing routine. The code is totally implemented in MATLAB, widely using MATLAB vector logic to efficiently deal with large arrays. The paper shows the validation of the code, which was performed with the data input and on the simulation scenarios suggested by the international benchmark of LTD simulators. In-train forces are computed with a LUT approach. A good agreement between the outputs produced by LTDPoliTO and the results from the other participants was obtained. Moreover, the code also proved to be a computationally fast and numerically stable tool in the four simulation scenarios, thus overcoming the inefficiencies of a previous model developed by the research team using the multibody software Simpack.

Simulations were run with the new code using piecewise linear functions describing the hysteretic mechanical impedance characteristic of the coupling elements, and the output results of this set of simulations were processed by the modal analysis post-processing routine. The natural frequencies calculated at the beginning of each simulation scenarios were in good agreement with the expectations. Moreover, the calculation of the system natural 
frequencies along the whole third simulation scenario showed a great variation of the largest system eigenfrequency, caused by changes in the deflection, relative speed and stiffness of all the train coupling elements. Therefore, when designing a heavy haul train configuration, great attention must be paid to these changes in the system eigenfrequencies, to avoid external forces to excite natural vibration modes. Moreover, the calculation of the train eigenfrequency evolution along a specific track can be extremely helpful for the selection of the proper sensors and transducers if on-track tests need to be performed to evaluate the train longitudinal dynamic behaviour.

The code validation process showed that the main goal of the activity was achieved and that the new LTDPoliTO code is a computationally fast and numerically stable tool for LTD simulations in both time and frequency domains.

Work is already in progress to adapt the code to the simulation of any user-defined train configuration built with any kind of wagon and locomotive. At the same time, simulations on the four benchmark trains are planned to be performed, investigating the influence of the position of the remote locomotives on the maximum tensile and compressive in-train forces, as a recent paper [34] shows that the traction and payload distribution along the train can significantly modify the maximum forces arising on the connection elements. Moreover, the code will be upgraded by adding a pneumatic module, able to consider the braking effort applied by the air brake system installed on freight trains. Concerning the modal analysis tool, a fitting equation or a white-box model will be developed to allow an easier calculation of the coupling element stiffnesses and to distinguish between the draft gear elastic and damping contributions.

\section{REFERENCES}

[1] Colin, C., Longitudinal Train Dynamics. Handbook of Railway Vehicle Dynamics, Taylor \& Francis: Boca Ratón, 2006.

[2] Massa, A., Stronati, L., Aboubakr, A.K., Shabana, A.A. \& Bosso, N., Numerical study of the noninertial systems: application to train coupler systems. Nonlinear Dynamics, 68(1), pp. 215-233, 2012. https://doi.org/10.1007/s11071-011-0220-2.

[3] Serajian, R., Mohammadi, S. \& Nasr, A., Influence of train length on in-train longitudinal forces during brake application. Vehicle System Dynamics, 57(2), pp. 192-206, 2019. https://doi.org/10.1080/00423114.2018.1456667.

[4] Wu, Q., Spiryagin, M. \& Cole, C., Longitudinal train dynamics: an overview. Vehicle System Dynamics, 54(12), pp. 1688-1714, 2016. https://doi.org/10.1080/00423114.201 6.1228988.

[5] Chen, D., Derailment risk due to coupler Jack-Knifing under longitudinal buff force. Proceedings of the Institution of Mechanical Engineers, Part F: Journal of Rail and Rapid Transit, 224(5), pp. 483-490, 2010. https://doi.org/10.1243/09544097jrrt363.

[6] Zhu, T., Yang, B., Yang, C., Xiao, S., Yang, G. \& Yang, B., The mechanism for the coupler and draft gear and its influence on safety during a train collision. Vehicle System Dynamics, 56(9), pp. 1375-1393, 2018. https://doi.org/10.1080/00423114.2017.1413198.

[7] Cole, C., Spiryagin, M., Wu, Q. \& Sun, Y.Q., Modelling, simulation and applications of longitudinal train dynamics. Vehicle System Dynamics, 55(10), pp. 1498-1571, 2017. https://doi.org/10.1080/00423114.2017.1330484.

[8] Cole, C., Improvements to wagon connection modelling for longitudinal train simulation. Conference on railway engineering proceedings: engineering innovation for a competitive edge, 1998. 
[9] Bosso, N., Gugliotta, A. \& Zampieri, N., Study of adhesion and evaluation of the friction forces using a scaled roller-rig. 5th World Tribology Congress, WTC 2013, pp. 2640-2643, 2014.

[10] Bosso, N. \& Zampieri, N., Real-time implementation of a traction control algorithm on a scaled roller rig. Vehicle System Dynamics, 51(4), pp. 517-541, 2013. https://doi.org/ 10.1080/00423114.2012.750001.

[11] Bosso, N., Gugliotta, A. \& Zampieri, N., RTCONTACT: An efficient wheel-rail contact algorithm for real-time dynamic simulations. 2012 Joint Rail Conference, pp. 195-204, 2012. https://doi.org/10.1115/jrc2012-74044.

[12] Bosso, N. \& Zampieri, N., A Novel Analytical Method to Calculate Wheel-Rail Tangential Forces and Validation on a Scaled Roller-Rig. Advances in Tribology, 2018. https://doi.org/10.1155/2018/7298236.

[13] Spiryagin, M., Wu, Q. \& Cole, C., International benchmarking of longitudinal train dynamics simulators: benchmarking questions. Vehicle System Dynamics, 55(4), pp. 450-463, 2017. https://doi.org/10.1080/00423114.2016.1270457.

[14] Wu, Q., Spiryagin, M., Cole, C., Chang, C., Guo, G., Sakalo, A., et al., International benchmarking of longitudinal train dynamics simulators: results. Vehicle System Dynamics, 56(3), pp. 343-365, 2018. https://doi.org/10.1080/00423114.2017.1377840.

[15] Wu, Q., Cole, C., Luo, S. \& Spiryagin, M., A review of dynamics modelling of friction draft gear. Vehicle System Dynamics, 52(6), pp. 733-758, 2014. https://doi.org/10.1080 /00423114.2014.894199.

[16] Pogorelov, D., Yazykov, V., Lysikov, N., Oztemel, E., Arar, O.F. \& Rende, F.S., Train 3D: the technique for inclusion of three-dimensional models in longitudinal train dynamics and its application in derailment studies and train simulators. Vehicle System Dynamics, 55(4), pp. 583-600, 2017. https://doi.org/10.1080/00423114.2016.1273532.

[17] Bosso, N., Gugliotta, A. \& Zampieri, N., A mixed numerical approach to evaluate the dynamic behavior of long trains. Procedia Structural Integrity, 12, pp. 330-343, 2018. https://doi.org/10.1016/j.prostr.2018.11.083.

[18] Bosso, N. \& Zampieri, N., Long train simulation using a multibody code. Vehicle System Dynamics, 55(4), pp. 552-570, 2017. https://doi.org/10.1080/00423114.2016.1 267373.

[19] Cantone, L., Crescentini, E., Verzicco, R. \& Vullo, V., A numerical model for the analysis of unsteady train braking and releasing manoeuvres. Proceedings of the Institution of Mechanical Engineers, Part F: Journal of Rail and Rapid Transit, 223(3), pp. 305-317, 2009. https://doi.org/10.1243/09544097jrrt240.

[20] Cantone, L., TrainDy: the new Union Internationale des Chemins de Fer software for freight train interoperability. Proceedings of the Institution of Mechanical Engineers, Part F: Journal of Rail and Rapid Transit, 225(1), pp. 57-70, 2011.

[21] Chang, C., Guo, G., Wang, J. \& Ma, Y., Study on longitudinal force simulation of heavy-haul train. Vehicle System Dynamics, 55(4), pp. 571-582, 2017. https://doi.org/1 0.1080/00423114.2016.1269183.

[22] Wu, Q. \& Cole, C., Computing schemes for longitudinal train dynamics: sequential, parallel and hybrid. Journal of Computational and Nonlinear Dynamics, 10(6), 2015. https://doi.org/10.1115/1.4029716.

[23] Wu, Q., Cole, C., Spiryagin, M., Wang, Y., Ma, W. \& Wei, C., Railway air brake model and parallel computing scheme. Journal of Computational and Nonlinear Dynamics, 12(5), 2017. https://doi.org/10.1115/1.4036421. 
[24] Wu, Q., Spiryagin, M. \& Cole, C., Parallel computing scheme for three-dimensional long train system dynamics. Journal of Computational and Nonlinear Dynamics, 12(4), 2017. https://doi.org/10.1115/1.4035484.

[25] Uyulan, C. \& Arslan, E., Simulation and time-frequency analysis of the longitudinal train dynamics coupled with a nonlinear friction draft gear. Nonlinear Engineering, 9(1), pp. 124-144, 2020. https://doi.org/10.1515/nleng-2020-0003.

[26] Bosso, N., Magelli, M. \& Zampieri, N., Development and validation of a new code for longitudinal train dynamics simulation. Proceedings of the Institution of Mechanical Engineers, Part F: Journal of Rail and Rapid Transit, 2020. https://doi. org/10.1177/0954409720923497.

[27] Bosso, N., Magelli, M. \& Zampieri, N., Long train dynamic simulation by means of a new in-house code. WIT Transactions on the Built Environment, Vol 199, WIT Press, 2020, ISSN: 1743-3509.

[28] Rochard,B.P.\&Schmid,F., A review of methods to measure and calculate train resistances. Proceedings of the Institution of Mechanical Engineers, Part F: Journal of Rail and Rapid Transit, 214(4), pp. 185-199, 2000. https://doi.org/10.1243/0954409001531306.

[29] Maksym Spiryagin, Colin Cole, Yan Quan Sun, Mitchell McClanachan, Valentyn Spiryagin \& McSweeney, T., Longitudinal Train Dynamics. Design and Simulation of Rail Vehicles, CRC Press: Boca Raton, 2014.

[30] Bosso, N., Magelli, M., Rossi Bartoli, L. \& Zampieri, N., The influence of resistant force equations and coupling system on LTD simulations. Proceedings of the Institution of Mechanical Engineers, Part F: Journal of Rail and Rapid Transit (Preprint), 2021.

[31] Zhang, Z., Li, G., Chu, G., Zu, H. \& Kennedy, D., Compressed stability analysis of the coupler and buffer system of heavy-haul locomotives. Vehicle System Dynamics, 53(6), pp. 833-855, 2015. https://doi.org/10.1080/00423114.2015.1023318.

[32] Shampine, L.F. \& Reichelt, M.W., The MATLAB ode suite. SIAM Journal of Scientific Computing, 18(1), pp. 1-22, 1997. https://doi.org/10.1137/S1064827594276424.

[33] Shampine, L.F., Gladwell, I., Shampine, L. \& Thompson, S., Solving ODEs with MATLAB, Cambridge university press, pp., 2003.

[34] Krishna, V.V., Jobstfinke, D., Melzi, S. \& Berg, M., An integrated numerical framework to investigate the running safety of overlong freight trains. Proceedings of the Institution of Mechanical Engineers, Part F: Journal of Rail and Rapid Transit, 235(1), pp. 47-60, 2021. https://doi.org/10.1177/0954409720905203. 